JOURNAL DE PHYSIQUE

Colzoque C2, supplément au $n^{\circ} 3$, Tome 44, mars 1983

page $\mathrm{C} 2-27$

\title{
DEGENERATE FREQUENCY MIXING IN SATURABLE AMPLIFIERS
}

J. Reintjes, B.L. Wexler, N. Djeu and J.L. Walsh

Laser Physics Branch, U.S. Naval Research Laboratory, Washington, D.C. 20375, U.S.A.

Résumé - Nous avons ëtudiê la conjugaison de phase par le mélange de quatre ondes dégénèrées dans les amplificateurs saturés pour une variété de gêométries d'action réciproque et de combinaisons de polarisation. Les résultats théoriques sont comparés avec les mesures dans un amplificateur XeCl. Un degré de rëflexions expérimental, s'éîevant jusqu'à 100 est observé.

\begin{abstract}
We have studied phase conjugation by degenerate frequency mixing in saturable amplifiers for various interaction geometries and polarization combinations. Theoretical results are compared with measurements in a $\mathrm{XeC} 1$ amplifier. Experimental reflectivities as high as 100 are observed.
\end{abstract}

\section{INTRODUCTION}

Phase conjugation through degenerate frequency mixing (DFM) in single-photon resonant media has attracted considerable attention. Because of the resonant enhancement of the nonlinear susceptibility these media have the potential for high phase-conjugate reflectivities. While phase conjugation in saturable absorbers has been analyzed in several approximations in the literature /1-8/ and has been studied experimentally in some detail /9-16/. DFM in saturable amplifiers has been studied less extensively in the literature /17-19/. Saturable amplifiers have some advantages for phase conjugation. For example, they are automatically matched to the corresponding laser frequency, providing a phase conjugating medium in regions of the spectrum in which other materials are hard to find. Secondly, they have the potential for providing high reflectivities at low pump powers if advantage can be taken of the gain of the medium. In this paper, we shall describe some of our theoretical and experimental results on DFM in two level saturable amplifiers.

We consider a two-level system with a transition frequency w10, a transition dipole moment, $\mu_{10}$, a dephasing time $T_{2}$ and a lifetime. $T_{1}$. We assume that there is a steady state population inversion in the medium that is maintained in the absence saturating fields by a suitable pump source. The small signal amplitude gain of coefficient for this system is given by

$$
\alpha=\frac{\left|\mu_{10}\right|^{2} T_{2}\left(N_{1}-N_{0}\right)}{\pi(1+i \delta)}=\frac{\alpha_{0}}{1+i \delta}
$$

where $\delta=(\omega 10-\omega) T_{2}$ is the fractional detuning, and the square of the saturation field is 


$$
A_{s}{ }^{2}=\frac{\hbar^{2}\left(1+\delta^{2}\right)}{\left|\mu_{10}\right|^{2} T_{1} T_{2}}
$$

We consider a nonlinear interaction involving four waves as shown in Fig. 1. Strong pump waves $E_{1}$ and $E_{2}$ propagate along the $+z^{\prime}$ and $-z^{\prime}$ directions respectively. A probe wave $E_{3}$ propagates along direction $z$, at a small angle $\theta$, to $z^{\prime}$. The fourth wave $E_{4}$ is generated in the interaction and propagates in a direction opposite to that of $E_{3}$. We will treat the nonlinear interaction of these waves due to saturation of the gain and we will include the effects of the variation of the pump wave amplitudes as they propagate through the medium.

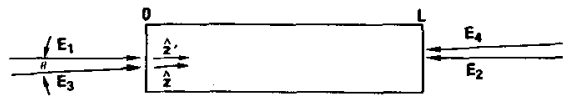

Fig. 1 - Schematic of experimental arrangement for DFM interaction.

The electric fields of the waves are given by

$$
\begin{aligned}
& E_{1}\left(z^{\prime}, t\right)=\frac{1}{2}\left(E_{1}\left(z^{-}\right) e^{-i \omega t}+c_{0} c_{0}\right) \\
& =\frac{1}{2}\left(A_{1}\left(z^{-}\right) e^{\left.-i\left(\omega t-k z^{-}\right)+c_{0} c_{0}\right)}\right. \\
& E_{2}\left(z^{-}, t\right)=\frac{1}{2} \quad\left(E_{2}\left(z^{-}\right) e^{-i \omega t}+c_{.} c_{0}\right) \\
& =\frac{1}{2}\left(A_{2}\left(z^{-}\right) e^{\left.-i\left(\omega t+k z^{-}\right)+c_{0}\right)}\right. \\
& E_{3}(z, t)=\frac{1}{2} \quad\left(E_{3}(z) e^{-i \omega t}+c . c .\right) \\
& =\frac{1}{2}\left(A_{3}(z) e^{\left.-i(\omega t-k z)+c_{0} \cdot c_{0}\right)}\right.
\end{aligned}
$$




$$
\begin{aligned}
E_{4}(z, t) & =\frac{1}{2}\left(E_{4}(z) e^{-i \omega t}+c_{0} c_{0}\right) \\
& =\frac{1}{2}\left(E_{4}(z) e^{\left.-i(\omega t+k z)+c_{0} c_{0}\right)}\right.
\end{aligned}
$$

and the nonlinear polarization due to gain saturation is giver by

$$
P(z)=\frac{1}{2}\left(\frac{-i \lambda \alpha \sum E_{i}}{4 \pi^{2}\left(1+\left|\sum E_{i}\right|^{2} / A s^{2}\right)}+c \cdot c \cdot\right)
$$

where $\alpha$ is the small signal amplitude gain coefficient and $E_{j}$ is the optical field of the ith wave as defined in equation 1. The component of the nonlinear polari$z$ ation that drives each wave can be obtained by taking the appropriate spatial Fourier component of each of the terms in equation 4 . The contribution of the various terms in the total polarization to a specific Fourier component depends on the relation of the polarization of the pump and probe waves, on the value of the angle $\theta$ and on the relative amplitudes of the various waves. We will illustrate the contributions of the various types of terms in the polarization by considering the Fourier components that drive waves $E_{1}$ and $E_{4}$.

We consider first a situation in which all the waves are polarized parallel to one another and the probe and generated field amplitudes are small compared to those of the pump waves. In this situation we can make an expansion of the denominator in equation 4 giving for the total polarization

$$
P(z)=-\frac{i \lambda \alpha \quad \sum E_{i}}{8 \pi^{2} D^{-}}\left(1-\frac{\left(E_{3}+E_{4}\right)\left(E_{1}^{*}+E_{2}^{*}\right)+\left(E_{3}^{*}+E_{4}^{*}\right)\left(E_{1}+E_{2}\right)}{D^{-}}+\right.\text {c.c. }
$$

where

$$
D^{-}=1+\left.\left|A_{1} e^{i k z^{\prime}+A_{2}} e^{-\left.i k z^{-}\right|^{2}}=1+\right| A_{1}\right|^{2}+\left|A_{2}\right|^{2}+2 A_{1} A_{2} \cos \left(2 k z^{\circ}+\phi 1-\phi_{2}\right)
$$

where $\phi_{1}$ and $\phi_{2}$ are the phases of $A_{1}$ and $A_{2}$, respectively.

The nonlinear polarization amplitude with the same fourier component as $E_{1}$ is given by

$$
P_{1}=-\frac{i \lambda \alpha}{4 \pi^{2}}\left(\frac{1}{2 \pi} \int \frac{A_{1}\left(z^{-}\right)}{D^{-}\left(z^{-}\right)} d z^{-}+\frac{1}{2 \pi} \int \frac{A_{2}(z) e^{-i 2 k z^{-}}}{D^{-}\left(z^{-}\right)} d z^{-}\right)
$$


In the slowly varying envelope approximation the integrals can be evaluated over a distance of the order of a wavelength and the field amplitudes can be treated as constants. Normally, the second term would not contribute to the nonlinear polarization for $E_{1}$ since it would not be phased matched. In the present situation, however, the intereference term in the demoninator couples the forward and backward waves and gives a non-zero contribution of this term to the polarization for $E_{1}$.

The nonlinear polarization amplitude with the same Fourier component as $E_{4}$ has two types of terms:

$$
\begin{aligned}
& P_{4}=\frac{-i \lambda \alpha}{8 \pi^{3}}\left(\int \frac{A_{4}(z)}{D^{-}\left(z^{\prime}\right)} d z-\int \frac{A_{4}\left(\left|A_{1}\right|^{2}+\left|A_{2}\right|^{2}\right) / A_{s^{2}}+2 A_{1} A_{2} A_{3}^{*} / A_{5}{ }^{2}}{D^{-2}\left(z^{\prime}\right)} d z^{-}\right) \\
& -\frac{i \lambda \alpha}{8 \pi^{2}}\left(\int \frac{A_{1} e^{i k\left(z^{-}+z\right)}}{D^{-}} d z+\int \frac{A_{2} e^{i k\left(z-z^{-}\right)}}{D^{-}} d z+\int \frac{A_{3} e^{i 2 k z}}{D^{-}} d z\right. \\
& -\int \frac{\left(A_{3}^{*} A_{1}^{2} / A_{s}{ }^{2}+A_{4} A_{1} A_{2}^{*} / A_{s}{ }^{2}\right) e^{i 2 k z^{\prime}}}{D^{-2}} d z \\
& -\int \frac{\left(A_{4}^{*} \cdot A_{1}^{2} / A_{s}{ }^{2}+A_{3} A_{1} A_{2}{ }^{4} / A_{s}{ }^{2}\right) e^{i 2 k\left(z^{-}+z\right)}}{D^{-2}} d z \\
& -\int \frac{\left(A_{4}^{*} A_{2}{ }^{2} / A_{s}{ }^{2}+A_{3} A_{1}^{*} A_{2} / A_{s} s^{2}\right) e^{i 2 k\left(z-z^{\prime}\right)}}{D^{-2}} d z \\
& -\int \frac{\left(2 A_{4}{ }^{*} A_{1} A_{2} / A_{s}{ }^{2}+A_{3}\left(\left|A_{1}\right|^{2}+\left|A_{2}\right|^{2}\right) / A_{s}{ }^{2}\right) e^{i 2 k z}}{0^{-2}} d z \\
& \left.-\int \frac{\left(A_{4} A_{1}^{*} A_{2} / A_{s}{ }^{2}+A_{3}{ }^{*} A_{2}{ }^{2} / A_{s}{ }^{2}\right) e^{-i 2 k z^{\prime}}}{D^{-2}} d z\right)
\end{aligned}
$$

The first set of these terms are those that are normally associated with the polarization of the backward wave. The remaining terms with different exponential variations in the numerator would normally be non-phase matched and would not contribute to the polarization of the backward wave. In the present situation they can be coupled to the backward wave by the interference term in the denominator. If, however, the field amplitudes vary slowly over a distance $L_{c} \approx \pi \lambda / \theta^{2}$ and the interaction length is larger than $L_{c}$ then the contribution of these terms 
will average to zero over the entire interaction length. These terms will, however, contribute to the nonlinear polarization if the probe and pump waves are collinear, i.e. if $\theta=0$.

Using similar considerations for the other two waves and evaluating the integrals in equations 7 and 8 we arrive at the following equations for the four waves:

$$
\begin{aligned}
& \frac{d A_{1}}{d z}=\bar{\alpha}_{1} A_{1} \\
& \frac{d A_{2}}{d z}=\bar{\alpha} A_{2} \\
& \frac{d A_{3}}{d z}=\bar{\alpha}_{3} A_{3}-\overline{\kappa A_{4}}{ }^{*} \\
& \frac{d A_{4}}{d z}=-\bar{\alpha}_{3} A_{4}+\bar{\kappa} A_{3}^{*}
\end{aligned}
$$

where

$$
\begin{aligned}
& \bar{\alpha}_{1}=\alpha_{0} \quad\left[\frac{1}{D}-\frac{1}{2 A_{1}^{2}(z) / A_{s}^{2}}\left(1-\frac{1+A_{1}^{2}(z) / A_{s}{ }^{2}+A_{2}{ }^{2}(z) / A_{s}{ }^{2}}{D}\right)\right] \\
& \bar{\alpha}_{2}=\alpha_{0}\left[\frac{1}{0}-\frac{1}{2 A_{2}{ }^{2}(z) / A_{s}{ }^{2}}\left(1-\frac{1+A_{1}{ }^{2}(z) / A_{s}{ }^{2}+A_{2}{ }^{2}(z) / A_{s}{ }^{2}}{D}\right)\right] \\
& \bar{\alpha}_{3}=\alpha_{0}\left(\frac{1+A_{1}^{2}(z) / A_{s}^{2}+A_{2}^{2}(z) / A_{s}^{2}}{D^{3}}\right) \\
& =\bar{\alpha}_{3}^{\prime}(z)+\overline{i \alpha_{3}}{ }^{\prime \prime}(z) \\
& \bar{k}=\alpha_{0}\left(\frac{2 A_{1}(z) A_{2}(z) / A_{s}{ }^{2}}{D^{3}}\right) \\
& 0=\left[1+2\left(A_{1}^{2}(z)+A_{2}^{2}\right) / A_{s}^{2}+\left(A_{1}^{2}(z)-A_{2}^{2}(z)\right)^{2} / A_{s}{ }^{2}\right]^{1 / 2}
\end{aligned}
$$

In deriving these equations we have assumed that the detuning is zero, that all the fields are real and that $\theta$ is small enough that the variation of the pump fields in the direction $z$ is effectively the same as the variation in the direction $z^{\prime}$. 


\section{THEORETICAL RESULTS}

We have examined the DFM reflectivity, defined as $I_{3}(0) / I_{4}(0)$, for several interaction geometries in the weak probe wave limit. In Fig. 2 we show calculated values of the reflectivity as a function of the incident pump wave intensity relative to the saturation intensity for the situation in which the incident intensity of the two pump beams is the same. The curves are parameterized by the small signal intensity gain length product $2 \alpha_{0} L$. The qualitative behavior of all the curves is similar. The reflectivity increases with the pump intensity at low pump intensity, reaches a peak and then decreases at higher pump intensities as the nonlinearity saturates. The peak reflectivity increases as $\alpha_{0} \mathrm{~L}$ increases, while the optimum incident pump intensity decreases with increasing $\alpha_{0} L$. For sufficiently large $\alpha_{0}$ L the peak reflectivity can be substantially larger than unity while the optimum incident pump intensity can be well below the saturation intensity. This qualitative behavior is similar to that predicted for saturable absorbers $/ 1 /$. However for saturable absorbers the peak reflectivity is generally below unity, even for reasonably Targe values of $\alpha_{0} L$ and the optimum incident pump intensity becomes greater than the saturation intensity as $\alpha_{0} L$ increases $/ 3 /$.

The numerical solutions are also compared to the predictions of an analytic result which can be obtained if the pump waves are assumed to be constant throughout the interaction $/ 1 /$. These solutions are shown as dashed curves in Fig. 2. The two types of solution are in fair agreement at low values of $\alpha_{0} \mathrm{~L}$. As $\alpha_{0} L$ increases, however, the peak reflectivity and the optimum pump intensity predicted by the constant pump approximation become too large. oscillation is predicted by the constant pump approximation for values of $2 \alpha_{0} L$ above about 7 . Such oscillations are not present in the numerical solutions that take into account the variation of the pump wave intensity in the nonlinear medium.

In many degenerate frequency mixing experiments it is convenient to obtain the backward pump wave by retroreflecting the transmitted forward pump wave at the end of the nonlinear medium. We show the results of calculations for such an arrangement in Fig. 3. Here we have assumed that the intensity gain Tength product is 8 . The various curves are for pump mirror reflectivities (RM) as indicated. For the value of $\alpha_{0} L$ used for these calculations the peak DFM reflectivity increases as the value of RM decreases from unity and the curves become more sharply peaked as a function of incident pump inten-

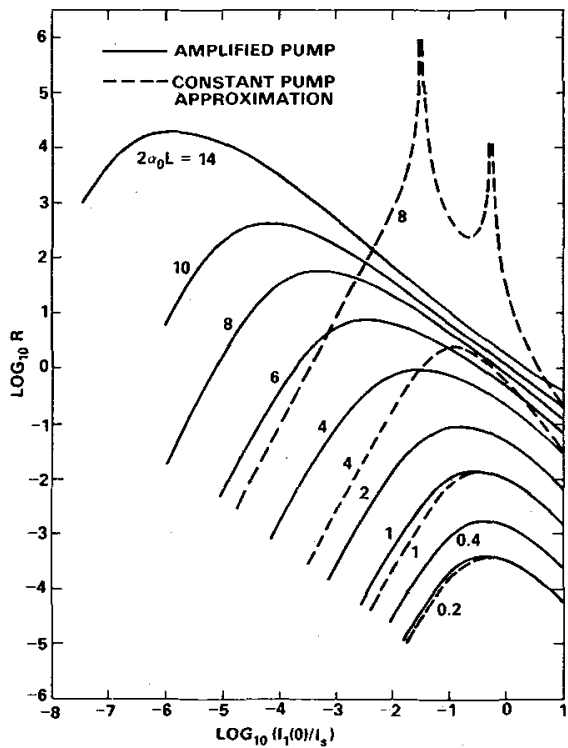

Fig. 2 - OFM reflectivity for various intensity gain length products for equal incident pump intensities. sity. Eventualiy, however, for sufficiently low reflectivity of the pump mirror, the peak DFM reflectivity begins to decrease again. The dashed curve represents the DFM reflectivity for the situation in which the incident pump wave intensities are equal. For incident pump wave intensities above the optimum value these results indicate that the optimum DFM reflectivity occurs when the reflectivity of the pump 
wave mirror is such that the reflected pump wave intensity $12(L)$ is the same as the incident pump wave intensity $I_{1}(0)$. For incident pump wave intensities below the optimum value this condition does not hold and the optimum reflectivity can occur for unequal incident pump wave intensities.

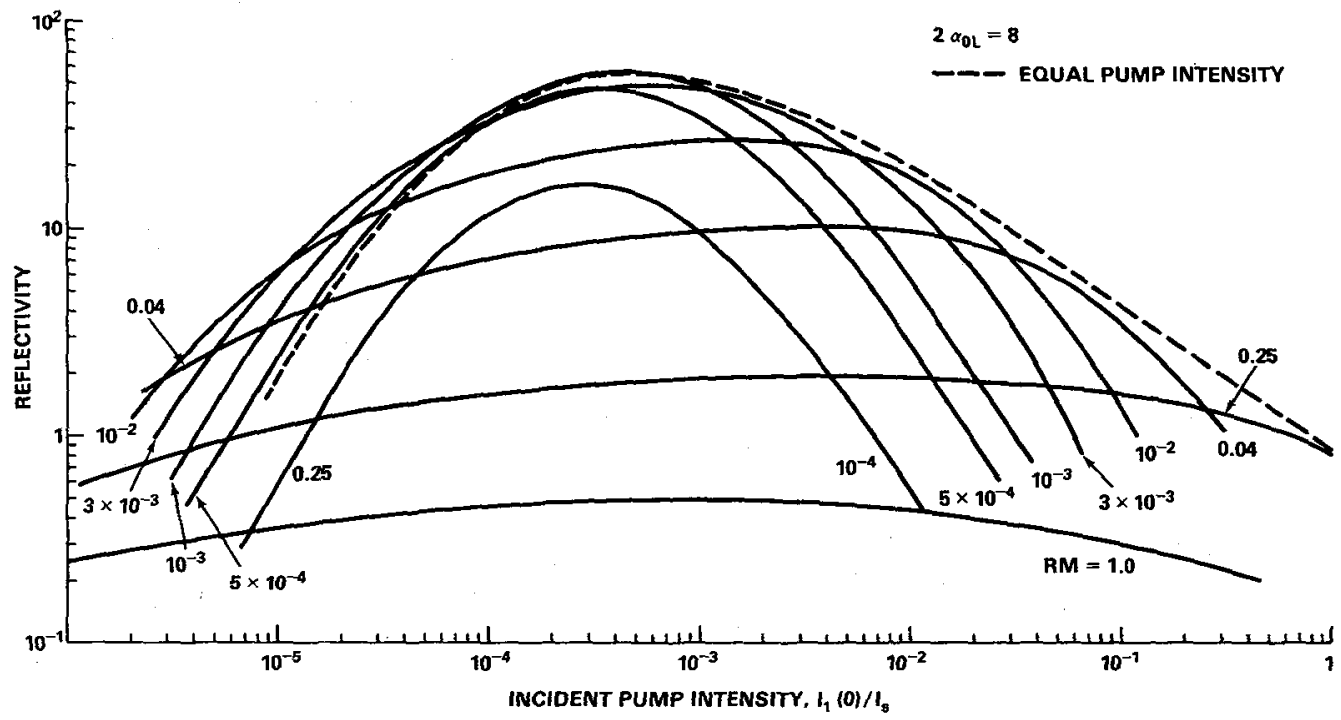

Fig. 3 - DFM reflectivity for a geometry in which the backward pump wave is obtained by retroreflecting the forward pump wave after the amplifier with a mirror of the reflectivity indicated on the curves. The dashed curve is for equal incident pump intensities.

Because of the requirement of a non-zero angle between the pump and probe waves, the probe and conjugate waves will experience amplification as they propagate to and from the nonlinear interaction region unless the crossing region is at the front of the amplifier. If the crossing region is at the rear of the amplifier we can anticipate that there will be an optimum division of the available length of the amplifier into a gain region and a nonlinear interaction region. We show in Fig. 4 calculated DFM reflectivies for pump wave mirrors of reflectivities of 1.0 and 0.04 for various interaction lengths in an amplifier with a total gain length product of 8. The interaction region is assumed to start at the back of the amplifier. In these curves only the reflectivity due to the DFM interaction is considered. Again the curves show a peak in the reflectivity at an optimum value of the incident pump intensity. As is to be be expected the DFM reflectivity increases as the interaction length increases. At small interaction lengths the DFM reflectivity obtained with $R M=1$ is greater than that obtained with $R M=0.04$. At long interaction lengths, however, this relation reverses and the DFM reflectivity that is obtained with the $4 \%$ reflector in the pump waves exceeds that which is obtained with the unity reflector.

The final set of curves in Fig. 4 (dash-dot) shows the DFM reflectivity for a geometry in which the two pump waves are orthogonally polarized and the probe wave is polarized parallel to one pump wave, while the reflected wave is polarized parallel to the other. For these curves we have assumed that the mirror reflectivity in the pump wave was unity. In this geometry the nonlinear interaction 
involves only one of the two spatial gratings formed by the DFM process. Here we see that the reflectivity for the orthogonal polarizations is quite close to that obtained with parallel polarizations for small pump wave intensity, but that it falls off faster with increasing pump wave intensity.

The total reflectivity for this system, taking into account the amplification of the probe and conjugate waves in the non-interacting part of the amplifier is shown in Fig. 5. Again the curves are parameterized by the fraction of the amplifier length that is involved in the nonlinear interaction. Curves for two different reflectivities for the pump wave mirror are shown. In each case there is an optimum length for the nonlinear interaction, which, for these values, is a small fraction of the total length. For example the optimum nonlinear interaction length for the unity reflectivity mirror in the pump waves occurs near $1 / 8$ of the total length, while the optimum fraction for the $4 \%$ mirror is near $1 / 4$ of the total length. The peak reflectivity obtained with $\mathrm{RM}=1$ is about $10^{4}$ while that for the 4\% mirror is somewhat smaller. In calculating these values we have assumed that the probe and conjugate wave intensities are small enough that they do not saturate the amplifier on their way to and from the nonlinear interaction region.

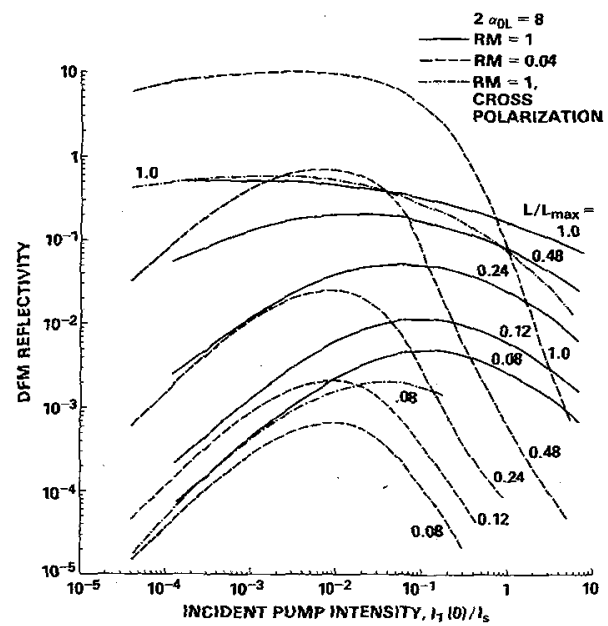

Fig. 4 - DFM reflectivity for various nonlinear interaction lengths, indicated as a fraction of the total length on each curve. Solid curves are for $R M=1.0$, dashed curves are for $R M=$ 0.04 . Chain curves are for $R M=1.0$ and orthogonal polarization.

Finally we have examined the DFM reflectivity when the probe wave is not necessarily small compared to the pump wave intensity. We have assumed for this interaction that the pump waves are orthogonally polarized, while the probe wave is polarized parallel to the backward pump wave and the conjugate wave is parallei to the forward pump wave. Under these conditions the equations for the four field amplitudes are:

$$
\begin{aligned}
& \frac{d A_{1}}{d z}=\bar{\alpha}_{1} \cdot A_{1}+\overrightarrow{k_{1}} \cdot A_{4} \\
& \frac{d A_{2}}{d z}=-\overline{\alpha_{1}}-A_{2}-\overline{\kappa_{1}}-A_{3} \\
& \frac{d A_{3}}{d z}=\overline{\alpha_{1}}-A_{3}+\overline{\kappa_{1}}-A_{2}
\end{aligned}
$$




$$
\frac{d A_{4}}{d z}=-\bar{\alpha}_{1}-A_{4}-\overline{k_{1}}-A_{1}
$$

where

$$
\begin{aligned}
& \vec{\alpha}^{-}=\frac{\alpha_{0}}{D^{\prime}} \\
& \bar{\kappa}_{1}{ }^{-}=\frac{\alpha_{0}}{2\left(A_{1} A_{4}+A_{2} A_{3}\right)}\left[1-\frac{\left[\left|A_{i}\right|^{2}\right.}{D^{\prime}}\right] \\
& D^{-}=\left[\left(1+\left[\left|A_{i}\right|^{2}\right)^{2}-4\left(A_{1} A_{4}+A_{2} A_{3}\right)^{2}\right]^{1 / 2}\right.
\end{aligned}
$$

The results of a calculation for $2 \alpha_{0} \mathrm{~L}=8$ and $R M=1$ are shown in Fig. 6 . The curve labeled 0 is the DFM reflectivity in the weak probe limit and is the same as that shown in Fig. 4. The other two curves are for larger values of $\mathrm{I}_{3}(0)$. At high pump wave intensities the OFM reflectivity approaches that of the weak probe wave limit, while for lower incident pump intensities the DFM reflectivity falls off quite rapidly, with the point of deviation moving to lower pump wave intensities as the intensity of the incident probe wave intensity decreases. It is also evident that the weak probe wave approximation can be satisfied even under conditions in which the incident probe wave intensity is greater than the incident pump wave intensity.

\section{EXPERIMENTAL RESULTS}

We have examined some of these properties by studying DFM reflectivity in a $\mathrm{XeC} 1$ amplifier. The experimental geometry is shown in Fig. 7. The amplifier was an $x$-ray preionized electric discharge device with a total length of $80 \mathrm{~cm}$ and an intensity gain-length product of 8 . The nonlinear interaction region was in the back of the amplifier extending about $7 \mathrm{~cm}$ from the rear window. The pump and probe waves were derived from a separate XeCl oscillator-amplifier. The output of this system was spectrally narrowed to about $0.1 \mathrm{~cm}^{-1}$ to ensure that the coherence length of the pump and probe waves was greater than nonlinear interaction length. The intensities of the incident pump waves the incident probe wave and the backward generated wave were measured as a function of the incident pump wave intensity for values of RM of 1.0 and 0.04 . The measured reflectivity included the effects of gain of the probe and generated waves in the non-interacting part of the amplifier. In the course of the measurements the incident pump wave intenstiy varied over the range $10^{-3} \mathrm{I}_{\mathrm{s}}$ to $0.5 \mathrm{I}_{\mathrm{s}}$. The total reflectivity was generally in the range 6 to 30 with values as high as 100 being observed. We have compared our results with the calculations described earlier by removing the gain experienced by the two waves in the noninteracting part of the amplifier. In this analysis it was evident that for the parameters of our experiment the probe and generated waves would saturate the amplifier, so the saturated gain was used rather than the unsaturated gain. In addition we have reduced the calculated reflectivity by a factor 2 to account for 
the fact that we were in the regime in which the backward grating was washed out by Doppler motion $/ 5 \%$. With these considerations the experimental DFM measurements are compared with the theoretical ones in Fig. 8. Generally the agreement is within a factor 2. The residual error could arise from failure of the weak probe wave approximation in the experiment.

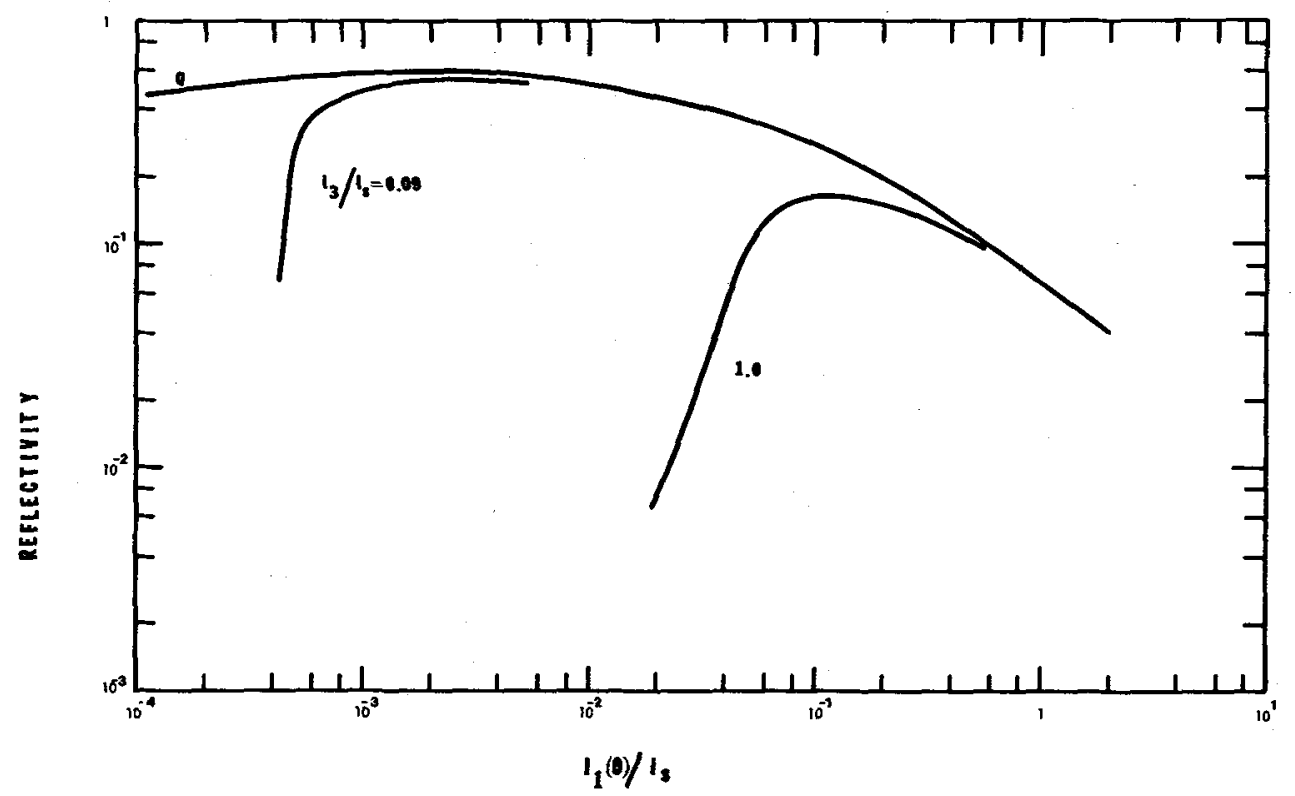

Fig. 6 - DFM reflectivity for orthogonal polarizations for various values of the incident probe wave intensity.

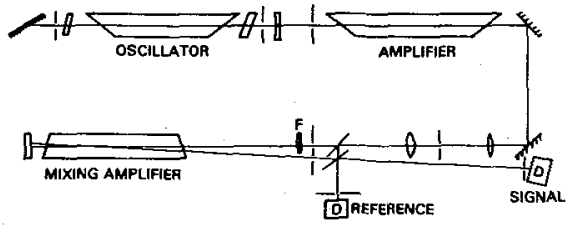

Fig. 7 - Experimental arrangement for observing DFM reflectivity in XeC 1 .

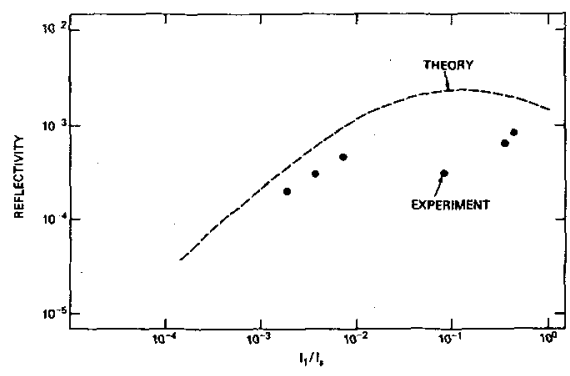

Fig. 8 - Comparison of theoretical DFM reflectivity and experimental measurements, corrected for the effects of gain outside of the nonlinear interaction length. 


\section{REFERENCES}

1. Abrams, R. L. and Lind, R. C., Opt. Comm. 2 (1978) 94; Erratum, Opt. Comm. $\underline{3}(1978) 205$.

2. Apanesevich, P. A., Afanas'ev A. A., and Zhvavyi, S. P., Sov. J. Quant. Elect. 10 (1980) 906.

3. Brown, W. P., Conference on Lasers and Electro-0ptics, Washington, D. C., 1981, Paper FP1.

4. Dunning, G. J. and Steel, D. G., IEEE J. Quant. Elect. QE-18 (1982) 3.

5. Wandzura, S. M., Opt. Lett. 4 (1979) 208.

6. Nilsen, J. and Yariv, A., J. Opt. Soc. Amer. 71 (1981) 180.

7. Bloom, D. M., Liao, P. F. and Economou, N. P., Opt. Lett. 2 (1978) 58.

8. Agrawal, G. P. and Flytzanis C., IEEE J. Quant. ETect. QE-17 (1981) 374.

9. Liao, P. F., Bloom, D. M., aand Economou, N. P., App7. Phys. Lett. 32 (1978) 813.

10. Liao, P. F. and Bloom, D. M., Opt. Lett. 3 (1978) 4.

11. Grischowsky, D., Shiren, N. S., and Bennett, R. J., Appt. Phys. Lett. 33 (1978) 805.

12. Lind, R. C., Steel, D. G., Klein, M. B., Abrams, R. L., Giuliano, C. R., and Jain, R. K., Appl. Phys. lett. 34 (1979) 457.

13. Moses, E. I. and Wu F. Y., Opt. Lett. $\underline{5}$ (1980) 64.

14. Steel, D. G., Lind, R. C., and Lam,J, F., Phys, Rev. A23, 19812513.

15. Tocho, J. 0., Sibbett, W., and Bradley, D. J., Opt. Comm. 34, (1980) 122.

16. Silberberg, Y. and Bar-Joseph, I., Opt. Comm. 39, (1981) 265.

17. Tomita, A., App1. Phys. Lett. 34 (1979) 463.

18. Fisher, R. A. and Feldman, B. J., Opt. Lett. 4 (1979) 140.

19. Bunkin, F. V., Savaranskii, V. V., and Shafeev, G. A., Sov. J. Quant. Elect. 11 (1981) 810 . 